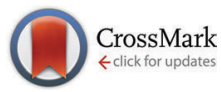

Cite this: Phys. Chem. Chem. Phys., 2016, 18, 9715

Received 22nd December 2015 Accepted 13th March 2016

DOI: $10.1039 / c 5 c p 07910 a$

www.rsc.org/pccp

\section{The effects of symmetry and rigidity on non-adiabatic dynamics in tertiary amines: a time-resolved photoelectron velocity-map imaging study of the cage-amine $A B C O$}

\author{
Liv B. Klein, ${ }^{a}$ Thorbjørn J. Morsing, ${ }^{a}$ Ruth A. Livingstone, ${ }^{b}$ Dave Townsend ${ }^{\text {cd }}$ and \\ Theis I. Sølling*a
}

\begin{abstract}
The non-adiabatic relaxation dynamics of the tertiary cage-amine azabicyclo[2.2.2]octane (ABCO, also known as quinuclidine) have been investigated following 3p Rydberg excitation at $201 \mathrm{~nm}$ using femtosecond time-resolved photoelectron imaging (TRPEI). The aim of the study was to investigate the influence of the rigid and symmetric cage structure found in $A B C O$ on the general non-adiabatic relaxation processes commonly seen in other tertiary aliphatic amines (TAAs). Our data is compared with TRPEI results very recently obtained for several structurally less rigid TAA systems [J. O. F. Thompson et al., Chem. Sci., 2016, 7, 1826-1839] and helps to confirm many of the previously reported findings. The experimental results for $\mathrm{ABCO}$ in the short-time $(<1 \mathrm{ps})$ regime strongly support earlier conclusions suggesting that planarization about the $\mathrm{N}$-atom is not a prerequisite for efficient $3 p-3 s$ internal conversion. Additionally, individual photoelectron peaks within our ABCO data show no temporal shifts in energy. As confirmed by our supporting quantum mechanical calculations, this demonstrates that neither internal conversion within the $3 p$ manifold or significant conformational re-organization are possible in the $A B C O$ system. This result therefore lends strong additional support to the active presence of such dynamical effects in other, less conformationally restricted TAA species, where photoelectron peak shifts are commonly observed. Finally, the extremely long (>1 ns) 3s Rydberg state lifetime seen in ABCO (relative to other TAA systems at similar excitation energies) serves to illustrate the large influence of symmetry and conformational rigidity on intramolecular vibrational redistribution processes previously implicated in mediating this aspect of the overall relaxation dynamics.
\end{abstract}

\section{Introduction}

We recently reported a time-resolved photoelectron imaging (TRPEI) study of $N, N$-dimethylisopropylamine (DMIPA), $N, N$-dimethylpropylamine (DMPA) and $N$-methylpyrrolidine (Mpyr). ${ }^{1}$ For all three of these tertiary aliphatic amine (TAA) systems, two distinct dynamical timescales were observed following $200 \mathrm{~nm}$ excitation - "ultrafast" (i.e. $<1 \mathrm{ps)} \mathrm{components} \mathrm{and} \mathrm{a} \mathrm{much}$ longer lived (38-160 ps) process. In the case of Mpyr, initial excitation predominantly populates the lowest member of the $3 p$ Rydberg manifold, which then undergoes internal conversion directly to the $3 \mathrm{~s}$ state with an exponential decay time $\tau_{1}=400 \pm 30 \mathrm{fs}$.

\footnotetext{
${ }^{a}$ Department of Chemistry, University of Copenhagen, Universitetsparken 5, DK-2100 Copenhagen Ø, Denmark.E-mail: theis@kiku.dk

${ }^{b}$ Center for Free-Electron Laser Science, DESY, Notkestrasse 85, 22607 Hamburg, Germany

${ }^{C}$ Institute of Photonics \& Quantum Sciences, Heriot-Watt University, Edinburgh, EH14 4AS, UK

${ }^{d}$ Institute of Chemical Sciences, Heriot-Watt University, Edinburgh, EH14 4AS, UK
}

This is subsequently followed by conformational relaxation on the $3 \mathrm{~s}$ potential surface $\left(\tau_{2}=640 \pm 50 \mathrm{fs}\right)$, as revealed by a distinct energy shift in the $3 \mathrm{~s}$ photoelectron peak with time. In contrast, for DMPA and DMIPA, an energetic shift was observed in the photoelectron peaks associated with ionization from the $3 p$ manifold, prior to internal conversion. As the optically bright $3 \mathrm{p}$ state in these two cases is the one located in the middle of the $3 \mathrm{p}$ manifold, the shift was ascribed to some combination of inter-manifold internal conversion and fast conformational rearrangement $\left(\tau_{1}=130 \pm 20 \mathrm{fs}\right.$ and $\tau_{1}=300 \pm 30 \mathrm{fs}$, respectively), followed by the internal conversion $\left(\tau_{2}=580 \pm\right.$ $40 \mathrm{fs}$ and $\tau_{2}=480 \pm 40 \mathrm{fs}$ ). More generally, we note that the predominantly Rydberg character exhibited by the low-lying excited states of TAA systems potentially offers an extremely useful "workbench" for using photoionization to systematically study the general interplay between molecular structure, nonadiabatic dynamics and chemical function in great detail. This is a consequence of Rydberg state energies exhibiting high sensitivity to ion core geometry (the so-called "Rydberg fingerprint") ${ }^{2}$ 


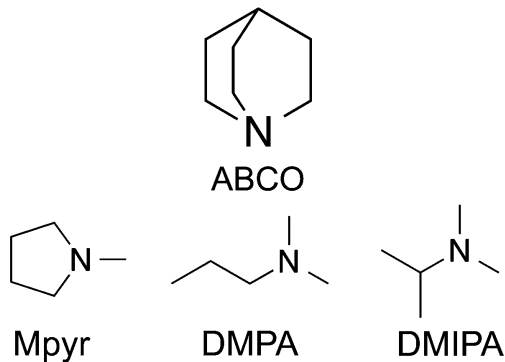

Fig. 1 Schematic structure of the cage-amine azabicyclo[2.2.2]octane (ABCO), shown together with the tertiary amines Mpyr, DMPA and DMIPA that were investigated previously in the recent work presented in ref. 1.

in combination with the inherently good spectral resolution afforded by a strong propensity for diagonal (i.e. $\Delta v=0$ ) transitions to the cation. This is in contrast to ionization of electronic states displaying predominantly valence character, where subtle changes in nuclear configuration may often be obscured due to broad and strongly overlapping ionization signatures from different electronic states.

In this paper, we report TRPEI experiments and supporting calculations on the cage-amine 1-azabicyclo[2.2.2]octane (ABCO, also known as quinuclidine), the structure of which is shown in Fig. 1 along with those of the recently investigated TAAs mentioned above. ABCO possess a rigid structure and a related lack of conformeric freedom but is otherwise a very similar system to the previously investigated TAAs (being a saturated tertiary amine of comparable size with very similar excited states and absorption spectroscopy). It is therefore a system well-suited to providing complimentary data to support our earlier findings. ${ }^{1}$ In addition, it provides an opportunity for investigation of the effects of the high symmetry of this species on the IVR processes thought to govern the decay of the 3s state. Previously ABCO has been the subject of considerable spectroscopic characterisation, with absorption bands, vibrational progressions, oscillator strengths and the electronic character of several excited states all having been investigated both experimentally ${ }^{3-6}$ and computationally. ${ }^{7,8}$ Photophysical properties such as fluorescence, ${ }^{9-11}$ excimer formation ${ }^{12,13}$ and ionization fragmentation ${ }^{14}$ have also been reported. The closely related 1,4-diazabicyclo[2.2.2]octane (DABCO) has been studied using time-resolved photoelectron spectroscopy, with the focus on the lonepair-lonepair interaction, ${ }^{15}$ which is not present in ABCO.

\section{Experimental}

The present experiment made use of a new velocity-map imaging (VMI) spectrometer recently constructed at the University of Copenhagen. Although somewhat different in overall appearance, many aspects of the design were based on an existing VMI instrument previously assembled at Heriot-Watt University that has been described in detail elsewhere. ${ }^{16}$ The Heriot-Watt machine was also used for the earlier studies of DMIPA, DMPA and Mpyr that are drawn upon extensively throughout this current work.

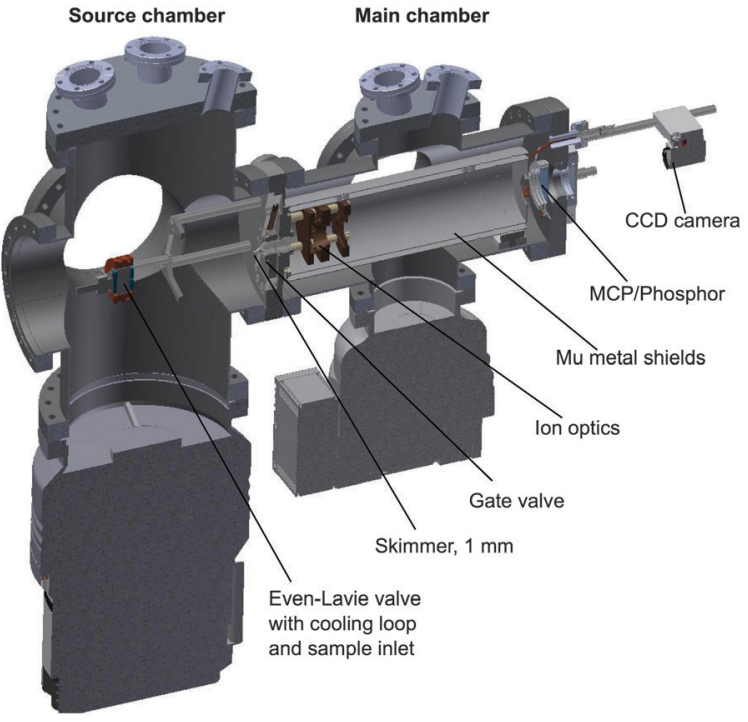

Fig. 2 Schematic cut-through of the photoelectron imaging spectrometer used in the present study. See the main text for additional information. Details such as vacuum-feedthroughs have been omitted for clarity. The mu-metal shields have outer diameters of $9 \mathrm{~mm}$ and $10.6 \mathrm{~mm}$ and are each $1 \mathrm{~mm}$ thick. The length of the flight tube is $28 \mathrm{~cm}$ from interaction region to detector.

A schematic overview of the new Copenhagen spectrometer is shown in Fig. 2. The vacuum system consists of separate source and main chambers with individual turbomolecular pumps (Pfeiffer vacuum, $1200 \mathrm{l} \mathrm{s}^{-1}$ and $400 \mathrm{l} \mathrm{s}^{-1}$ ). The chambers are connected by a $1.0 \mathrm{~mm}$ skimmer (Beam Dynamics Inc.) mounted on a translatable gate-valve assembly based on the design of Stolow, ${ }^{17}$ giving the convenient option of venting the source chamber to atmospheric pressure while maintaining ultrahigh vacuum in the main chamber. The ABCO sample was purchased from Sigma-Aldrich (99\% purity) and was introduced into the source chamber via an Even-Lavie pulsed supersonic molecular beam valve ${ }^{18}$ with a $150 \mu \mathrm{m}$ diameter conical nozzle operating at $1 \mathrm{kHz}$. The sample, a solid with a high melting point $\left(157-160^{\circ} \mathrm{C}\right)$, was held in a cartridge inserted into the back of the valve. The valve temperature was regulated at $55-65{ }^{\circ} \mathrm{C}$ in order to obtain a sufficient vapour pressure of the solid ABCO. No photoelectron signals associated with cluster formation were observed in the present data, as confirmed by switching the spectrometer into ion-detection mode. The carrier gas was helium at a pressure of 2.5 bars. After passing into the main chamber through the skimmer, the molecular beam was intersected perpendicularly by co-propagating UV pump $(201 \mathrm{~nm}$, $0.3 \mu \mathrm{J}$ per pulse) and probe $(268 \mathrm{~nm}, 1.3 \mu \mathrm{J}$ per pulse) laser beams. These were the fourth and third harmonics, respectively, produced from the fundamental $800 \mathrm{~nm}$ output of a $1 \mathrm{kHz}$ regeneratively amplified Ti:Sapphire laser system, which has been described elsewhere. ${ }^{19,20}$

The temporal delay between the pump and probe pulses was controlled using a linear translation stage (Newport, ILS150PP) and driver (Newport, ESP100), controlled by computer acquisition software. The laser beams were focused into the spectrometer through a $2.0 \mathrm{~mm} \mathrm{CaF}_{2}$ window using a concave spherical 
UV-enhanced aluminium mirror $(R=750 \mathrm{~mm})$. The interaction of the laser pulses with the molecular beam took place between the repeller and extractor electrodes of a mu-metal shielded electrostatic lens set-up optimized for photoelectron $\mathrm{VMI}^{21}$ and based on a previously used design. ${ }^{16,22}$ A $40 \mathrm{~mm}$ dual microchannel plate (MCP) detector coupled to a $\mathrm{P} 47$ phosphor screen (Photonis, APD 2 PS 40/12/10/12 I 46:1 P47) was positioned at the end of the short $(28 \mathrm{~cm})$ photoelectron flight tube. The voltage applied to the back of the MCP was gated using a high voltage pulser (DEI, PVX-4140). Photoelectrons mapped onto the detector were imaged using a monochrome USB 3.0 CCD camera (The Imaging Source, DMK23U618) with a $640 \times 480$ pixel array and an $8 \mathrm{~mm}$ megapixel fixed $f=1.4$ lens (Edmund Optics, M0814-MP2). The collected images were passed from the camera to a PC running a modified version of software previously developed at Heriot-Watt University. ${ }^{16}$

Data collection runs consisted of scanning the translation stage repeatedly between pump-probe delays of $-400 \mathrm{fs}$ to $+700 \mathrm{fs}$ in steps of $50 \mathrm{fs}$, then $100 \mathrm{fs}$ steps to $1 \mathrm{ps}$ followed by 22 exponentially increasing steps to $+250 \mathrm{ps}$. At each delay position sampled, time-invariant pump and probe alone signals were also collected for subsequent background subtraction. Data collection runs consisted of $\sim 20$ such scans, from which the images at each pump-probe delay were added together. Radius-to-energy calibration of the instrument was obtained using two-photon $268 \mathrm{~nm}$ non-resonant ionization of 1,3-butadiene, and the central wavelengths of both laser beams were precisely measured using a compact grating spectrometer (Ocean Optics, USB2000+). The experimentally determined temporal instrument response of $170 \pm 15$ fs was obtained directly inside the spectrometer from two-colour $\left(1+1^{\prime}\right)$ multiphoton ionization of 1,3-butadiene (assuming a small lifetime at these wavelengths of $\sim 20 \mathrm{fs}$ ). ${ }^{23-25}$

\section{Results}

\section{A. Time-resolved photoelectron spectra}

Fig. 3 presents photoelectron images resulting from $\left(1+1^{\prime}\right)$ ionization at selected pump-probe delays. Two sharp rings with significant anisotropy peaking along the laser polarization are present. This indicates that the excited states are of predominantly Rydberg character (as expected for a tertiary amine) since Rydberg states have a strong propensity for diagonal ionization $(\Delta v=0)$ and exhibit well-defined electronic orbital angular momentum. The left-hand half of each image shows the result following application of a rapid matrix inversion approach that has been described in detail elsewhere. ${ }^{16}$ Application of this inversion, along with appropriate energy calibration, permits time-resolved photoelectron spectra to be generated spanning the full range of pump-probe delay times, as shown in Fig. 4. It should be noted that the time axis is plotted on a mixed linear-logarithmic scale (switching at $1 \mathrm{ps)}$ ) and the energy axis is plotted in terms of Rydberg state binding energy (i.e. photoelectron kinetic energy subtracted from probe photon energy) to allow for ease of comparison with several previous TAA photoelectron studies. ${ }^{1,26,27}$ Two relatively narrow peaks are seen, one at $\sim 2.3 \mathrm{eV}$ decaying rapidly (i.e. in $<1 \mathrm{ps}$ ) and the other at $\sim 2.9 \mathrm{eV}$ that has a delayed onset from zero pump-probe delay and subsequently decays on a very extended timescale. Based on several previous photoelectron studies of tertiary amine systems at comparable excitation wavelengths, ${ }^{1,26,28-30}$ the lower and higher binding energy peaks may be attributed to ionization from an initially prepared $3 \mathrm{p}$ state and the $3 \mathrm{~s}$ state (populated subsequently via internal conversion), respectively.

An important feature of the spectra obtained in our previous study of more flexible TAA systems was the small $(\sim 0.1 \mathrm{eV})$ binding energy shifts observed in some of the photoelectron peaks within the first picosecond following excitation. This effect was visible in the form of a distinct shift in peak positions seen in $2 \mathrm{D}$ intensity plots of Rydberg binding energy $v s$. pumpprobe delay - especially when the intensity was presented on a logarithmic scale. An equivalent spectrum is presented in Fig. 5 for the case of ABCO. In this instance, neither of the two main spectroscopic features show any significant spectral shift in time. It should be noted that there is also a much weaker feature at slightly higher binding energies with respect to the 3s state (and following the same temporal evolution). This is likely due to a very small propensity for some non-diagonal (i.e., $\Delta v \neq 0$ ) ionization

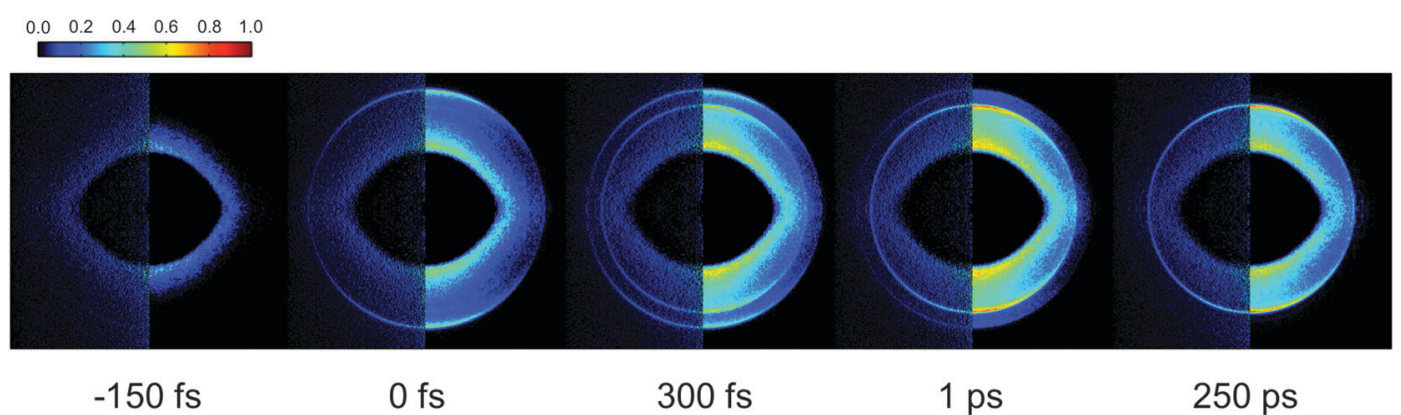

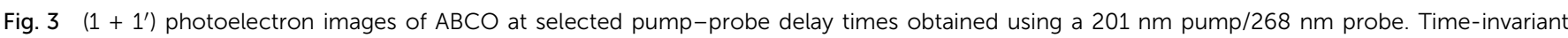

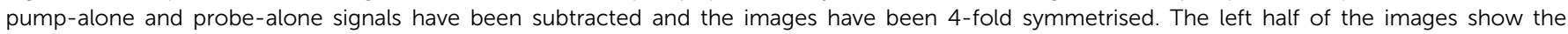

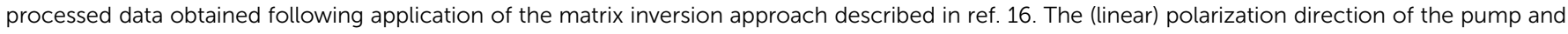

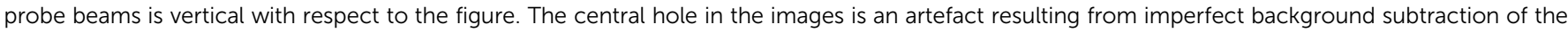

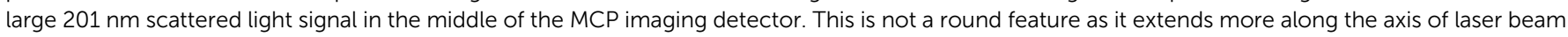
propagation. 


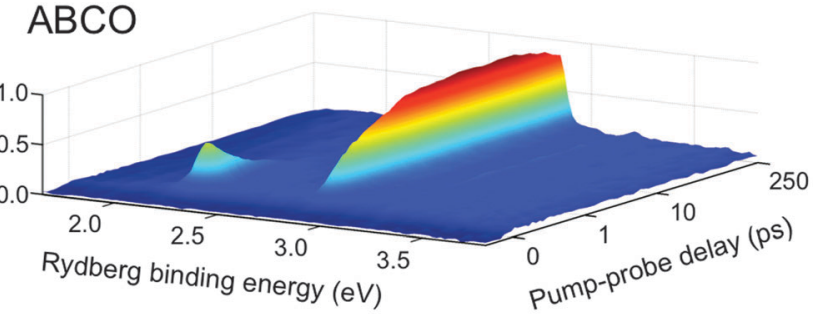

Fit

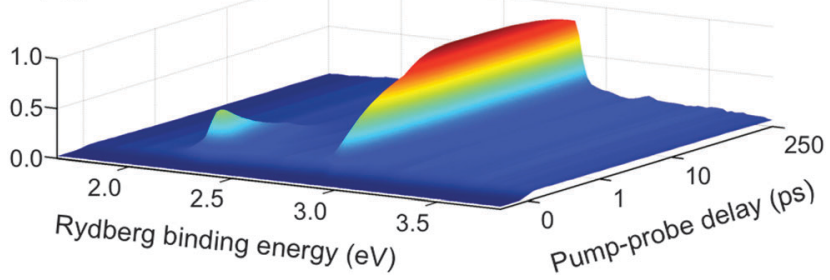

Fig. 4 3D time-dependent photoelectron spectrum obtained using a $201 \mathrm{~nm}$ pump/268 nm probe. For clear display of the dynamics over all temporal ranges, the time axis is linear to +1 ps and then logarithmic beyond this point. Also shown is the fit to the data. The residual (i.e. the fit minus the raw data) displays no observable features on the intensity scale used.

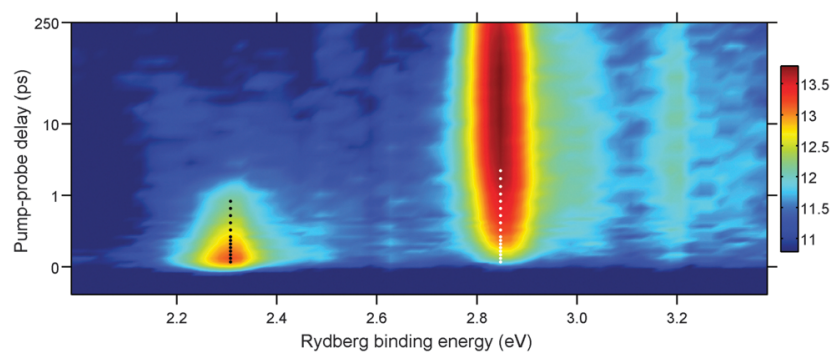

Fig. 5 2D time-dependent photoelectron spectrum obtained using a $201 \mathrm{~nm}$ pump/268 nm probe. As in Fig. 4, the time axis is linear to $+1 \mathrm{ps}$ and then logarithmic beyond this point. The intensity colour map is presented on a natural logarithmic scale based on the output directly obtained from the imaging CCD camera. Dot overlays denote centre positions of the main peak features obtained from Lorentzian fits performed at selected pump-probe delay times.

from the $3 \mathrm{~s}$ state involving a high-frequency vibrational mode (most probably a C-H stretch).

The time-dependence of the photoelectron data was examined by undertaking a sequential global fit using a LevenbergMarquardt routine, as previously used for the analysis of TRPEI data obtained for other TAA systems. ${ }^{1,31}$ The 2D data $S(E, \Delta t)$ are described using the following:

$$
S(E, \Delta t)=\sum_{i=1}^{n} A_{i}(E) \cdot P_{i}(\Delta t) \otimes g(\Delta t)
$$

Here $g(\Delta t)$ denotes the experimentally determined Gaussian instrument response function and $A_{i}(E)$ is the decay associated photoelectron spectrum of the $i$ th data channel with a time dependent population $P_{i}(\Delta t)$ defined as follows:

$$
P_{i}(\Delta t)=\mathrm{e}^{-\Delta t / \tau_{i}} \cdot\left(1-\mathrm{e}^{-\Delta t / \tau_{i-1}}\right)
$$

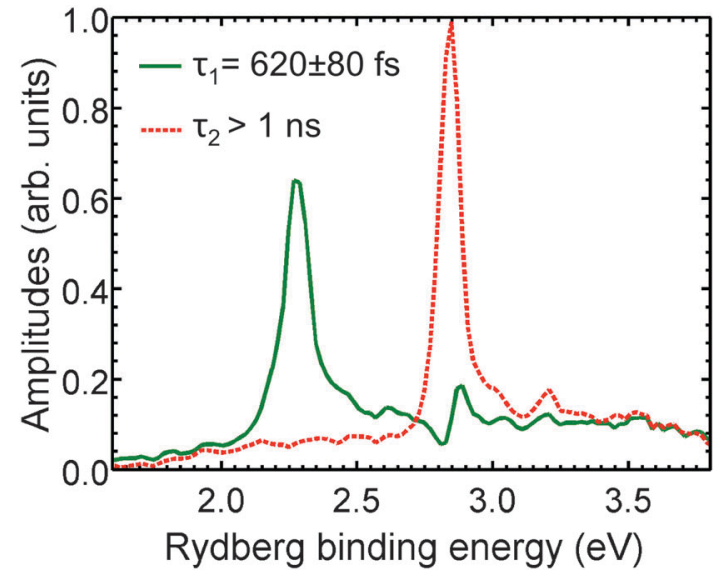

Fig. 6 Decay associated spectrum (DAS) obtained from a sequential twostep global exponential fit to the data presented in Fig. 4. For additional details see the main text. The quoted uncertainties are $1 \sigma$ values and the data is partitioned into $0.02 \mathrm{eV}$ energy bins.

This approach yields a set of decay associated spectra (DAS) plotting the relative amplitude of each fit component as a function of Rydberg binding energy. The ABCO data was fitted with two sequentially decaying exponential functions which we label using their respective time constants $\tau_{1}$ and $\tau_{2}$. The sequential nature of the fit means that $\tau_{2}$ grows in with the decay of $\tau_{1}$. The DAS plots obtained from the fit are shown in Fig. 6. The initial component originating from zero pump-probe delay $\left(\tau_{1}=620 \pm 80 \mathrm{fs}\right.$ ) has amplitude at Rydberg binding energies close to $2.3 \mathrm{eV}$. As discussed above (and as also confirmed by the theoretical work described later) this may be assigned to ionization from a $3 p$ state. For the similar cage-diamine DABCO, initial excitation at $251 \mathrm{~nm}$ has been found to give $\tau_{1}$ on a timescale of 1-3 ps, ${ }^{15,32}$ where the larger time constant may be explained by the lower excitation energy, as discussed in detail elsewhere. ${ }^{1}$ More generally, we note that DABCO (and other TAAs containing two amino groups) have been shown to exhibit additional interactions between the $\mathrm{N}$ atom lone pairs (with associated charge delocalization effects). ${ }^{15,33,34}$ This adds additional complexity to the dynamics and so a more detailed direct comparison between DABCO and ABCO is not considered further here. The second, much longer-lived fit component $\tau_{2}$ seen at $\sim 2.9 \mathrm{eV}$ arises from $3 \mathrm{~s}$ ionization once this state is populated via internal conversion. From the limited temporal range of our data we can only be certain that $\tau_{2}$ is greater than $1 \mathrm{~ns}$ and this is quoted as a lower-bound limit for the $3 \mathrm{~s}$ state lifetime. Notably, this is at least an order of magnitude longer that the $3 \mathrm{~s}$ lifetimes obtained for conformationally less rigid TAAs (Mpyr, DMPA and DMIPA) at comparable excitation wavelengths. ${ }^{1}$ In contrast to DMIPA, DMPA and Mpyr, adding a third time constant to the fit did not result in an improved or meaningful outcome. This is consistent with the lack of temporal evolution seen in the position of the ABCO photoelectron spectrum peaks.

\section{B. Photoelectron angular distributions}

Photoelectron images acquired using $\left(1+1^{\prime}\right)$ ionization with parallel linear polarizations yields photoelectron angular 
distributions (PADs) which can be described by the anisotropy parameters $\beta_{2}$ and $\beta_{4}$ as a function of excited state electron binding energy $E$ and pump-probe delay time $\Delta t:^{35,36}$

$$
I(E, \Delta t, \theta)=\frac{\sigma(E, \Delta t)}{4 \pi}\left[1+\beta_{2}(E, \Delta t) P_{2}(\cos \theta)+\beta_{4}(E, \Delta t) P_{4}(\cos \theta)\right]
$$

Here $\sigma(E, \Delta t)$ is the time-dependent electron energy distribution, the $P_{n}(\cos \theta)$ terms are the $n$ th-order Legendre polynomials, and $\theta=180^{\circ}$ is defined by a vertical line running fully through the centre of the images shown in Fig. 3. The temporal anisotropy evolution obtained from this fit is shown in Fig. 7 for the spectral features assigned to ionization from both the $3 \mathrm{p}$ and $3 \mathrm{~s}$ states. To ensure that symmetrisation of our images does not produce artefacts in the angular data, we also analyzed each quadrant of

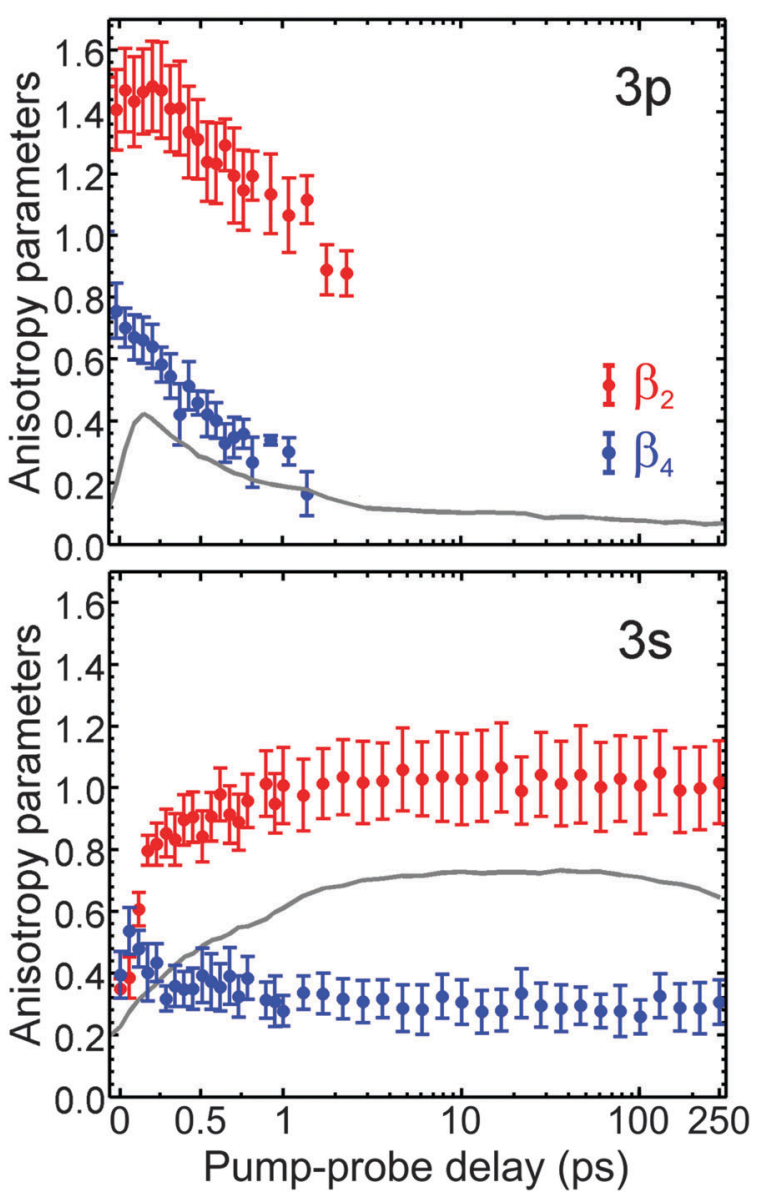

Fig. 7 Anisotropy parameters $\beta_{2}$ and $\beta_{4}$ as a function of pump-probe delay for $268 \mathrm{~nm}$ ionization from the $3 p$ and 3 s states. Plots show values averaged over the $0.3 \mathrm{eV}$ energy region spanned by the spectral feature associated with each state. The time axis is linear to +1 ps and then logarithmic. The data was partitioned into $0.02 \mathrm{eV}$ energy bins for the initial anisotropy fits (before averaging) and the error bars denote $1 \sigma$ values. Fits were performed over the angular region $5^{\circ} \leq \theta \leq 90^{\circ}$ to eliminate uncertainties from centre-line noise present in the Abel-inverted images (see Fig. 2). The solid line is the corresponding photoelectron signal within the same energy region. For the $3 p$ state, the plots for the anisotropy parameters are truncated at 1 ps as the signal level in the raw photoelectron data becomes so small that the fit becomes statistically meaningless. the un-symmetrized data independently, obtaining consistent results in all cases. For the $3 \mathrm{p}$ state, both $\beta_{2}$ and $\beta_{4}$ start out relatively high and then decay on a timescale comparable to the $\tau_{1}$ value obtained from the DAS fit described in the preceding section. For the $3 \mathrm{~s}$ state, $\beta_{2}$ and $\beta_{4}$ exhibit rapid evolution within the first picosecond following excitation and then remain constant for the rest of the experiment (with $\beta_{4}$ having a near-zero value). This overall behaviour is largely consistent with that seen in other TAA systems although, unlike in the case of DMIPA, DMPA and Mpyr, no long-time rise is observed for the anisotropy parameters associated with the $3 \mathrm{~s}$ state. This is possibly due to the much greater $3 \mathrm{~s}$ lifetime in $\mathrm{ABCO}$ and the limited range of pump-probe delays we were able to sample. Conducting an exploratory PAD measurement at a greatly extended $(\sim 1 \mathrm{~ns})$ pump-probe delay still failed to show any increase in either $\beta_{2}$ or $\beta_{4}$.

\section{Supporting calculations}

Vertical excitation energies and oscillator strengths for the four lowest singlet excited states of ABCO were evaluated using both density functional theory (CAM-B3LYP/aug-cc-pVDZ) and equation-of-motion coupled cluster (EOM-CCSD/aug-cc-pVDZ) approaches. Both methods appear to produce consistent output, as would be predicted on the basis of the more expansive comparative theoretical investigation employed in our previous recent TAA study. ${ }^{1}$ The results are shown in Table 1 and agree with previous experimental ${ }^{5}$ and theoretical ${ }^{8}$ ABCO investigations in terms of approximate relative oscillator strengths, while the energies from the present calculations are slightly higher (up to $0.3 \mathrm{eV}$ for the EOM-CCSD calculations). At both levels of theory employed, the ABCO excited state energies are very similar to those calculated with the same methods for DMIPA $(<0.1 \mathrm{eV}$ difference). ${ }^{1}$ The energy gap between the optically bright $3 p$ state and the $3 \mathrm{~s}$ state is also very similar in these two systems. As such, any differences observed in the excited state lifetimes of ABCO and DMIPA may (as a first approximation) be rationalized in terms of structural factors rather than significantly differing levels of internal vibrational excitation - as expanded upon later in the Discussion. All corresponding states lie slightly $(\sim 0.25 \mathrm{eV})$ higher in energy in DMPA and Mpyr. ${ }^{1}$ From the orbital coefficients returned by the calculations, all states appear very "pure" in terms of their Rydberg character in the vertical excitation region. The high symmetry of ABCO results in degeneracy of the two lowest $3 p_{x, y}$ states. This degeneracy means that excitation to either

Table 1 Singlet excitation energies and oscillator strengths of $A B C O$ calculated using CAM-B3LYP/aug-cc-pVDZ and EOM-CCSD/aug-cccpVDZ. The calculations were performed on a minimum energy geometry optimized at the B3LYP/aug-cC-PVDZ level. The two lowest 3p states are degenerate, and the assignments are based on the principle axis of symmetry being the $z$-axis for the molecule

\begin{tabular}{llllll}
\hline & \multicolumn{2}{c}{ CAM-B3LYP } & & \multicolumn{2}{c}{ EOM-CCSD } \\
\cline { 2 - 3 } & $E / \mathrm{eV}$ & & & $E / \mathrm{eV}$ & $f$ \\
\hline $3 \mathrm{~s} \leftarrow n_{\mathrm{N}}$ & 5.23 & 0.003 & & 5.12 & 0.005 \\
$3 \mathrm{p}_{x, y} \leftarrow n_{\mathrm{N}}$ & 5.85 & 0.024 & & 5.72 & 0.022 \\
$3 \mathrm{p}_{z} \leftarrow n_{\mathrm{N}}$ & 5.98 & 0.004 & & 5.81 & 0.004
\end{tabular}


one of these states will cause the molecule to undergo a JahnTeller distortion to minimize energy. This Jahn-Teller splitting is calculated to be very small $\left(<20 \mathrm{~cm}^{-1}\right)$, but results in the loss of $C_{3 \mathrm{v}}$ symmetry for the minimum energy structure for the lowestlying $3 p$ state. Geometry optimizations of the ground state (GS), the $3 \mathrm{~s}$ state and the lowest (and optically bright) 3p state minima were performed to explore this effect further. A complete-active space self-consistent field (CASSCF) approach was employed with a $(2,5)$ active space encompassing the nitrogen lone pair and four virtual orbitals (corresponding to the $3 \mathrm{~s}$ state and three $3 \mathrm{p}$ states). For $\mathrm{C}$ and $\mathrm{N}$ the aug-cc-pVDZ basis set was used with the addition of a set of diffuse 3 s functions $(\zeta=0.025$ and 0.021 , respectively) and $3 p$ functions $(\zeta=0.028$ for $\mathrm{C}$ and 0.023 for $\mathrm{N})$, similar to those used recently for the related system DABCO,${ }^{15}$ while cc-pVDZ was used for the $\mathrm{H}$ atoms. Calculations were performed using the ORCA program. ${ }^{37}$ The resulting geometries are shown in Fig. 8 and some illustrative structural parameters in the form of bond lengths and angles are listed in Table 2. Though there are small variations, possibly due to the specific convergence criteria on the geometry optimizations, the ground state and 3s state both
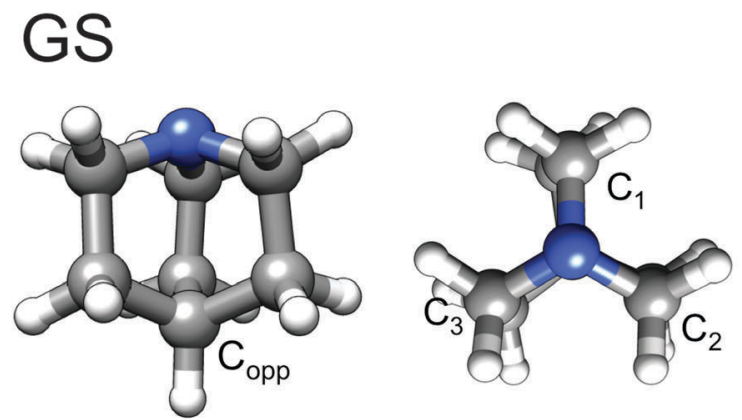

3s
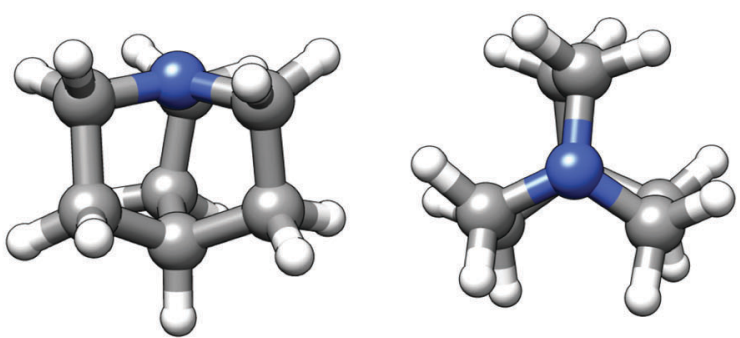

$3 p$
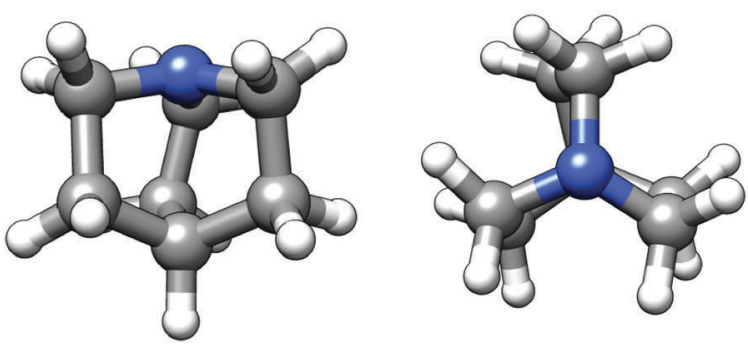

Fig. 8 CASSCF optimized minimum energy geometries of ABCO for the ground state (GS), 3s state and lowest 3p state. While the GS and 3s state have $C_{3 v}$ symmetry, the distortion in the $3 p$ geometry is caused by the Jahn-Teller effect. For selected structural parameters, see Table 2.
Table 2 Selected distances and angles from the CASSCF optimization for the ground state (GS), 3s and Jahn-Teller distorted lowest 3p state geometries. For further information see the main text

\begin{tabular}{llll}
\hline & GS & $3 \mathrm{~s}$ & $3 \mathrm{p}_{x}$ \\
\hline $\mathrm{N}-\mathrm{C}_{\mathrm{opp}} / \AA$ & 2.565 & 2.298 & 2.344 \\
$\mathrm{~N}-\mathrm{C}_{1} / \AA$ & 1.455 & 1.428 & 1.459 \\
$\mathrm{~N}-\mathrm{C}_{2} / \AA$ & 1.454 & 1.425 & 1.432 \\
$\mathrm{~N}-\mathrm{C}_{3} / \AA$ & 1.457 & 1.428 & 1.455 \\
$\mathrm{C}_{1}-\mathrm{N}-\mathrm{C}_{2} /{ }^{\circ}$ & 110.0 & 116.6 & 120.3 \\
$\mathrm{C}_{2}-\mathrm{N}-\mathrm{C}_{3} /{ }^{\circ}$ & 111.1 & 117.6 & 119.1 \\
$\mathrm{C}_{3}-\mathrm{N}-\mathrm{C}_{1} /{ }^{\circ}$ & 110.5 & 117.6 & 110.5
\end{tabular}

exhibit $C_{3 \mathrm{v}}$ symmetry. The Jahn-Teller distorted 3p state geometry shows one $\mathrm{N}-\mathrm{C}$ bond length significantly shorter and one $\mathrm{C}-\mathrm{N}-\mathrm{C}$ angle smaller than the other two. Due to the locked structure, neither the $3 \mathrm{~s}$ or the lowest $3 \mathrm{p}$ state minima exhibit a planar structure around the nitrogen atom, although the optimized geometries do exhibit a slightly greater $\mathrm{C}-\mathrm{N}-\mathrm{C}$ bond angle than in the ground state. As expected, no other stable conformations were located for any of the states.

Previously evolution along N-C stretching coordinates has been suggested as a critical nuclear motion for mediating both the $3 \mathrm{p} / 3 \mathrm{~s}$ internal conversion and the long-time $3 \mathrm{~s}$ relaxation dynamics of TAA systems. ${ }^{1}$ Potential energy cuts were therefore evaluated for the five lowest singlet states (including the ground state) along one of the three (identical) $\mathrm{N}-\mathrm{C}$ bond extension coordinates from a relaxed DFT (B3LYP) ground state surface scan. The nitrogen lone pair, two $\sigma$-orbitals and four virtual orbitals were used to make a $(6,7)$ active space, and the potential energy scans (shown in Fig. 9) were calculated as vertical excitation energies from the ground state geometry using state-averaged CASSCF/NEVPT2 ${ }^{38-40}$ (as implemented in ORCA). As was found previously for DMIPA using an EOMCCSD/aug-cc-pVDZ approach (and implied for DMPA and Mpyr), the 3s state remains bound, while one member of the

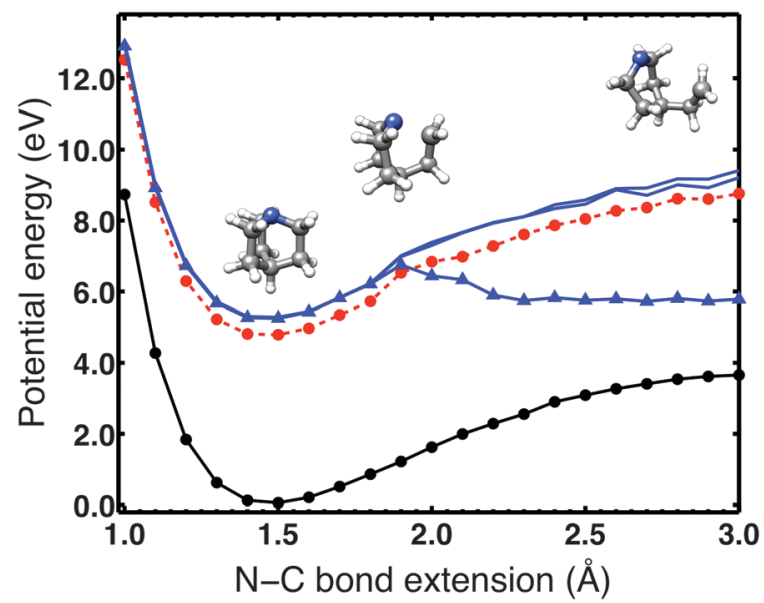

Fig. 9 CASSCF/NEVPT2 potential energy cuts for ABCO from a relaxed scan along an $\mathrm{N}-\mathrm{C}$ bond extension coordinate, showing the ground state (black), 3s state (red, dashed) and the three 3p states (blue, lowest marked with triangles). Also shown are the geometry at the ground state minimum, close to the expected conical intersection and at highly extended $\mathrm{N}-\mathrm{C}$ bond lengths. 
$3 \mathrm{p}$ manifold rapidly evolves to valence $\sigma^{*}$ character at a relatively small $\mathrm{N}-\mathrm{C}$ bond extension. We highlight that this is an intriguing result as in many small heteroatom-containing molecules it is now well-established that the evolution of $3 \mathrm{~s}$ to $\sigma^{*}$ character is a critical factor in driving the non-adiabatic photochemistry. ${ }^{41,42}$ The fact that instead a member of the $3 p$ manifold appears to evolve the valence character in TAA systems (rather than the $3 \mathrm{~s}$ state) is therefore a potentially important finding. At more extended $\mathrm{N}-\mathrm{C}$ distances the $3 \mathrm{p}\left(\sigma^{*}\right)$ state crosses the $3 \mathrm{~s}$ state, presumably giving rise a conical intersection along this coordinate. The existence of such a conical intersection has been verified computationally for DMIPA, ${ }^{1}$ and we assume by analogy that a similar crossing exists for $\mathrm{ABCO}$ given the overall similarities that exist between the experimentally observed dynamics in the two systems. However, there is no indication that the repulsive part of the $3 p\left(\sigma^{*}\right)$ potential should cross or get very near to the ground state.

\section{Discussion}

The time-dependent photoelectron spectrum presented in Fig. 4 shares the characteristics of previously reported TAAs, with the involvement of an initially excited 3p state decaying by internal conversion to the $3 \mathrm{~s}$ state, which then undergoes slower decay. ${ }^{1,29,30}$ The photoelectron peaks are located at very similar Rydberg binding energies to those of DMIPA, DMPA and Mpyr. Both of the observed peaks in the photoelectron spectra are narrow and exhibit a high degree of angular anisotropy, reflecting the predominantly Rydberg character of the excited states. Note that some anisotropy peaking perpendicular to the laser polarization is visible by eye in the outer ring (corresponding to ionization from the $3 \mathrm{p}$ state) in the images taken at $0 \mathrm{fs}$ and $300 \mathrm{fs}$ (see Fig. 3). This implies a significant $\beta_{4}$ contribution and this is also seen quantitatively at short-time pump-probe delays in the angular fits presented in Fig. 7. Such an observation indicates (at least at a "zero-order" atomic approximation for single photon ionization, where $\Delta l= \pm 1$ ) that the phase shift between the outgoing $\mathrm{s}$ and $\mathrm{d}$ waves is $\sim 60-90$ degrees. Some helpful examples illustrating the effect of partial wave phase on the appearance of PADs may be found in the work of Stolow and co-workers. ${ }^{43}$ This phase difference is somewhat larger than that inferred in our earlier TAA study $\left(<30^{\circ}\right)$ but is still smaller than would be expected on the basis of heuristic arguments using high- $n$ quantum defects to approximate the phase of the outgoing photoelectron partial waves $\left(>140^{\circ}\right)$. This possibly reflects either (i) a degree of mixing between the "s" and " $p$ " states (a process which certainly occurs at short delay times during internal conversion) and/or (ii) scattering of the outgoing electron off the non-spherical molecular ion core (i.e. the $\Delta l= \pm 1$ restriction is lifted). A more expanded discussion of these points (including the quantum defect approximation argument) may be found in ref. 1 . It is interesting to note that the larger apparent $s-d$ phase shift seen in ABCO (relative to DMIPA, DMPA and MPyr) - which seems to imply a situation closer to (although still clearly not) an "atomic-like" ionization picture - may be a consequence of reduced electron scattering due to the more "spherical" ion core geometry exhibited by the cage structure.

\section{A. Decay of the 3p state}

The supporting calculations presented in Section III.C confirm that $\mathrm{ABCO}$ does not possess multiple minimum energy conformers in any of its lowest singlet excited states due to the very rigid cage structure. In addition, the bright $3 \mathrm{p}$ state carrying the oscillator strength is the lowest-lying member of the manifold (see Table 1). This therefore eliminates the possibility of both inter-manifold internal conversion between the various $3 p$ states and also significant conformational rearrangement effects invoked previously to explain subtle temporal shifts seen in the photoelectron peaks in our recent study of small aliphatic tertiary amines ${ }^{1}$ and also in other TAA photoelectron studies. ${ }^{28-30}$ In keeping with this, the energetic maxima of both main features in the ABCO photoelectron spectrum presented in Fig. 5 appear invariant with time and display no shift in peak position, helping to confirm the previous TAA analysis. We also note that the calculated Jahn-Teller splitting discussed earlier is far too small $\left(<20 \mathrm{~cm}^{-1}\right)$ to give rise to an experimentally observable shift in the TRPEI signals associated with $3 p$ state ionization. The lifetime found for the 3p state of ABCO (620 \pm $80 \mathrm{fs}$ ) is similar to the overall 3p manifold lifetimes of DMIPA, DMPA and Mpyr $(560 \pm 40 \mathrm{fs}, 640 \pm 40 \mathrm{fs}$ and $400 \pm 30 \mathrm{fs}$, respectively). ${ }^{1}$ Since (in contrast to less conformationally rigid TAAs) the excited state minimum for ABCO is not planar about the $\mathrm{N}$ atom (as shown by our supporting calculations), these similar time constants strongly support our previous conclusion that planarization is not a prerequisite for efficient $3 \mathrm{p} / 3 \mathrm{~s}$ internal conversion. Looking at the anisotropy traces in Fig. 7, the initial non-zero $\beta_{4}$ value obtained for 3 s state ionization $(\sim 0.5)$ would not be expected for a state of predominantly s Rydberg character (since an s orbital inherently possesses no alignment and so cannot yield a $\beta_{4}$ term in the associated PAD upon ionization). As was the case for other TAAs, ${ }^{1}$ this is therefore thought to reflect the rapid non-adiabatic coupling to the $3 p$ state occurring at short times following the initial excitation, as the initial decay of the $\beta_{4}$ parameter matches the $3 \mathrm{p}$ state decay time.

\section{B. Decay of the 3s state}

The time constant $\tau_{2}$, describing the slow decay of the $3 \mathrm{~s}$ state in $\mathrm{ABCO}$ is determined to be in excess of $1 \mathrm{~ns}$. This is at least an order of magnitude larger than the observed lifetimes for the $3 \mathrm{~s}$ states of the three previously studied amines DMIPA, DMPA and Mpyr. ${ }^{1}$ As suggested by our calculations in Section III.C, and as also appears to be confirmed by comparing the band positions of the relevant absorption spectra,,4 the $3 \mathrm{~s}$ and optically bright $3 \mathrm{p}$ state of ABCO sit at very similar or slightly lower energies than these other systems. The corresponding $3 s-3 p$ energy gap is also therefore very similar. As such, the far longer $3 \mathrm{~s}$ lifetime seen in ABCO cannot simply be rationalized in terms of significant differences in internal vibrational excess energy (in fact the slightly larger size and associated higher density of states in ABCO compared to DMIPA etc. might reasonably be 
expected to result in a shorter 3 s lifetime under this rationale). Some other effect must be responsible and the obvious factor here is the conformational rigidity and symmetry of the ABCO structure. The $3 \mathrm{~s}$ state of $\mathrm{ABCO}$ is assigned to the $C_{3 \mathrm{v}}$ point group, which is a much higher symmetry than seen for any conformer of the $3 \mathrm{~s}$ state in the other TAA systems considered previously.

The greatly extended $3 \mathrm{~s}$ relaxation time in $\mathrm{ABCO}$ might imply that some of the processes previously ruled out in the case of conformationally less-rigid TAA systems may now play a role here. Fluorescence is the most likely option, but despite fluorescence lifetimes of more than $300 \mathrm{~ns}$ being reported at excitation wavelengths of both 236 and $249 \mathrm{~nm},{ }^{9}$ a dramatic drop in both quantum yield and fluorescence lifetime at lower excitation wavelengths would be expected, similar to what has been found for a range of smaller tertiary amines. ${ }^{44-46}$ An early multiphoton ionization study of ABCO has also shown the existence of a fast non-radiative decay channel for the Rydberg states. ${ }^{6}$ In our previous TRPEI study of other TAAs, an observed rise in the $\beta$ parameters at long pump-probe delay times was an important indicator in ruling out fluorescence decay. ${ }^{1}$ Such a rise has not been observed in the present data, and attempts to measure anisotropy values indicate that both $\beta$ parameters remain at constant value out to $1 \mathrm{~ns}$. Since we can only be sure that the $3 \mathrm{~s}$ lifetime is $>1 \mathrm{~ns}$, this is inconclusive and we cannot know with certainty if this rise will ultimately appear or not.

The interpretation used previously to explain the long-time decay of the 3s state in DMIPA, DMPA and Mpyr (38 ps, 130 ps and $160 \mathrm{ps}$, respectively) invoked intramolecular vibrational redistribution (IVR) on the $3 \mathrm{~s}$ potential energy surface as a prerequisite to facilitating re-crossing to the repulsive part of the $3 \mathrm{p}\left(\sigma^{*}\right)$ potential. The excited state wavepacket was then assumed to subsequently disappear rapidly from the experimental TRPEI observation window. The forms of the potential energy profiles along the $\mathrm{N}-\mathrm{C}$ stretch coordinate for $\mathrm{ABCO}$ (see Fig. 9) are very similar those evaluated previously for DMIPA. IVR is highly dependent on the coupling of vibrational modes and it has previously been demonstrated that symmetry hinders this process by imposing restrictions on which modes can couple, significantly slowing down the rate. ${ }^{47,48}$ As already highlighted, the minimum energy geometry of the $3 \mathrm{~s}$ state for $\mathrm{ABCO}$ is assigned to the $C_{3 \mathrm{v}}$ point group, a much higher degree of symmetry than that exhibited by any conformer of the TAA systems we have previously investigated. The higher symmetry in ABCO may therefore prevent effective coupling of vibrational modes, leading to a much longer IVR timescale. This could therefore explain the much slower decay of the $3 \mathrm{~s}$ state as the rate at which $\mathrm{ABCO}$ samples the correct geometry to undergo re-crossing to the repulsive part of the $3 \mathrm{p} / \sigma^{*}$ potential surface is greatly reduced. Another related factor that can influence IVR rates is torsional motion and the availability of "centres of flexibility" (bonds about which internal rotation occurs). ${ }^{49}$ The locked structure of ABCO means that internal rotations are extremely limited, giving no opportunity for IVR acceleration through torsion. The very long $3 \mathrm{~s}$ lifetime can in this way be at least partially explained by the unique aspects of ABCO's structure: high symmetry and conformational rigidity. We also note that at lower excitation energies $(<215 \mathrm{~nm})$, features in the absorption spectrum of $\mathrm{ABCO}$ associated with both the $3 \mathrm{~s}$ and $3 p$ states are much more structured than in many other TAA systems. ${ }^{3}$ This, more generally, also suggests (i) relatively long lifetimes in the $3 \mathrm{~s}$ state and (ii) that the $3 \mathrm{p}$ state lifetime increases considerably (compared to the situation at $200 \mathrm{~nm}$ ) - possibly indicating an energetic onset threshold for ultrafast $3 \mathrm{~s} / 3 \mathrm{p}$ internal conversion, which is clearly an interesting avenue for future investigation with the TRPEI approach.

From the present data we cannot determine the ultimate fate of the $3 \mathrm{~s}$ state for $\mathrm{ABCO}$ with certainty but by analogy with our other recent TAA investigations, it is possible that a re-crossing to the repulsive part of the $3 p\left(\sigma^{*}\right)$ surface may take place after extended time-scale IVR processes. If this were the case then the values of the anisotropy parameters should at some point begin to rise again (as was seen previously in DMIPA, DMPA and Mpyr), reflecting the "switch-on" of the required $3 \mathrm{~s}-3 \mathrm{p}$ coupling. However, it is also possible that for ABCO fluorescence now plays a part in the 3s state decay even at $200 \mathrm{~nm}$ excitation. To determine this definitively, further investigations are clearly necessary - for example fluorescence yield/lifetime at high excitation energy, time-resolved experiments giving the anisotropy parameters on a much more extended timescale, or additional high-level computational studies, all of which are beyond the current capabilities of our set-up.

\section{Conclusions}

The conformational rigidity and high symmetry of the ABCO cage structure makes it an interesting addition to the on-going study of the structural influence on the photophysics of simple tertiary amines. The mono-conformeric nature of $\mathrm{ABCO}$, and the fact that the optically bright $3 p$ state is the lowest-lying within the $3 \mathrm{p}$ manifold removes the two factors thought to give rise to observed temporal energy shifts of the peaks in the photoelectron spectra of more flexible TAAs. ${ }^{1,28-30}$ As a consequence of this, no temporal shifts in photoelectron peak position were observed in the present ABCO data. This serves to confirm the presence of inter-manifold internal conversion and extensive conformational changes in previously reported photoelectron data obtained from the less conformationally rigid systems Mpyr, DMPA and DMIPA. The similarity of the $3 p$ state lifetime in ABCO to that seen in DMIPA, DMPA and Mpyr indicates that achieving planarity about the $\mathrm{N}$ atom is not a critical prerequisite for non-adiabatic coupling between the $3 p$ and 3 s states. This is in agreement with computational results indicating that the internal conversion is mediated by a conical intersection between a $3 \mathrm{p}$ state evolving to valence character and the $3 \mathrm{~s}$ state along $\mathrm{N}-\mathrm{C}$ bond extension coordinates. A markedly longer $3 \mathrm{~s}$ lifetime (relative to less conformationally restricted TAAs) of more than a nanosecond also shows that the symmetry/rigidity of the ABCO system influences the final relaxation, most likely due to the slowing effect of symmetry on IVR processes. As a consequence of this very long lifetime, the final fate of the $\mathrm{ABCO} 3 \mathrm{~s}$ state is not clear from the limited 
temporal data range obtained this study but we tentatively suggest that it may be the same as other TAA systems - namely a re-crossing to the repulsive part of the $3 p\left(\sigma^{*}\right)$ surface. We hope that our findings will provide additional impetus to the on-going study of non-adiabatic dynamics in a broad range of TAA systems.

\section{Acknowledgements}

L. B. K. thanks the Danish Council for Independent Research | Natural Sciences (FNU) for PhD funding.

\section{References}

1 J. O. F. Thompson, L. B. Klein, T. I. Sølling, M. J. Paterson and D. Townsend, Chem. Sci., 2016, 7, 1826-1839.

2 J. L. Gosselin and P. M. Weber, J. Phys. Chem. A, 2005, 109, 4899-4904.

3 A. M. Halpern, J. L. Roebber and K. Weiss, J. Chem. Phys., 1968, 49, 1348-1357.

4 D. H. Parker and P. Avouris, Chem. Phys. Lett., 1978, 53, 515-520.

5 A. M. Weber, A. Acharya and D. H. Parker, J. Phys. Chem., 1984, 88, 6087-6089.

6 M. Fujii, N. Mikami and M. Ito, Chem. Phys., 1985, 99, 193-206.

7 P. Avouris and A. R. Rossi, J. Phys. Chem., 1981, 85, 2340-2344.

8 V. Galasso, Chem. Phys., 1997, 215, 183-190.

9 A. M. Halpern, J. Am. Chem. Soc., 1974, 96, 7655-7661.

10 A. M. Halpern, D. P. Gerrity, L. J. Rothberg and V. Vaida, J. Chem. Phys., 1982, 76, 102-107.

11 A. M. Halpern and A. Taaghol, J. Phys. Chem., 1989, 93, 144-149.

12 A. M. Halpern and E. Maratos, J. Am. Chem. Soc., 1972, 94, 8273-8274.

13 A. M. Halpern, J. Am. Chem. Soc., 1974, 96, 4392-4398.

14 D. H. Parker, R. B. Bernstein and D. A. Lichtin, J. Chem. Phys., 1981, 75, 2577-2582.

15 A. E. Boguslavskiy, M. S. Schuurman, D. Townsend and A. Stolow, Faraday Discuss., 2011, 150, 419-438.

16 R. A. Livingstone, J. O. F. Thompson, M. Iljina, R. J. Donaldson, B. Sussman, M. J. Paterson and D. Townsend, J. Chem. Phys., 2012, 137, 184304.

17 A. Stolow, J. Vac. Sci. Technol., A, 1996, 14, 2669-2670.

18 U. Even, J. Jortner, D. Noy, N. Lavie and C. Cossart-Magos, J. Chem. Phys., 2000, 112, 8068-8071.

19 N. Rusteika, K. B. Møller and T. I. Sølling, Chem. Phys. Lett., 2008, 461, 193-197.

20 N. Rusteika, R. Y. Brogaard, T. I. Sølling, F. M. Rudakov and P. M. Weber, J. Phys. Chem. A, 2009, 113, 40-43.

21 A. T. J. B. Eppink and D. H. Parker, Rev. Sci. Instrum., 1997, 68, 3477-3483.
22 E. Wrede, S. Laubach, S. Schulenburg, A. Brown, E. R. Wouters, A. J. Orr-Ewing and M. N. R. Ashfold, J. Chem. Phys., 2001, 114, 2629-2646.

23 W. Fuss, W. E. Schmid and S. A. Trushin, Chem. Phys. Lett., 2001, 342, 91-98.

24 F. Assenmacher, M. Gutmann, G. Hohlneicher, V. Stert and W. Radloff, Phys. Chem. Chem. Phys., 2001, 3, 2981-2982.

25 P. Hockett, E. Ripani, A. Rytwinski and A. Stolow, J. Mod. Opt., 2013, 60, 1409-1425.

26 J. L. Gosselin, M. P. Minitti, F. M. Rudakov, T. I. Sølling and P. M. Weber, J. Phys. Chem. A, 2006, 110, 4251-4255.

27 S. Deb, M. P. Minitti and P. M. Weber, J. Chem. Phys., 2011, 135, 044319.

28 M. P. Minitti and P. M. Weber, Phys. Rev. Lett., 2007, 98, 253004.

29 S. Deb, B. A. Bayes, M. P. Minitti and P. M. Weber, J. Phys. Chem. A, 2011, 115, 1804-1809.

30 J. D. Cardoza, F. M. Rudakov and P. M. Weber, J. Phys. Chem. A, 2008, 112, 10736-10743.

31 F. Rudakov, Y. Zhang, X. Cheng and P. M. Weber, Opt. Lett., 2013, 38, 4445-4448.

32 T. Seideman, Annu. Rev. Phys. Chem., 2002, 53, 41-65.

33 S. Deb, X. Cheng and P. M. Weber, J. Phys. Chem. Lett., 2013, 4, 2780-2784.

34 X. Cheng, Y. Zhang, S. Deb, M. P. Minitti, Y. Gao, H. Jónson and P. M. Weber, Chem. Sci., 2014, 5, 4394-4403.

35 K. L. Reid, Annu. Rev. Phys. Chem., 2003, 54, 397-424.

36 T. Suzuki, Annu. Rev. Phys. Chem., 2006, 57, 555-592.

37 F. Neese, see https://orcaforum.cec.mpg.de.

38 C. Angeli, R. Cimiraglia, S. Evangelisti and T. Leininger, J. Chem. Phys., 2001, 114, 10252-10264.

39 C. Angeli, R. Cimiraglia and J.-P. Malrieu, J. Chem. Phys., 2002, 117, 9138-9153.

40 C. Angeli, R. Cimiraglia and J.-P. Malrieu, Chem. Phys. Lett., 2001, 350, 297-305.

41 M. N. R. Ashfold, G. A. King, D. Murdock, M. G. D. Nix, T. A. A. Oliver and A. G. Sage, Phys. Chem. Chem. Phys., 2010, 12, 1218-1238 and references herein.

42 G. M. Roberts and V. G. Stavros, Chem. Sci., 2014, 5, 1698-1722 and references herein.

43 G. Wu, P. Hockett and A. Stolow, Phys. Chem. Chem. Phys., 2011, 18, 18447-18467.

44 C. D. Abbott, C. G. Cureton, J. Hara, S. Hirayama and D. Phillips, J. Photochem. Photobiol., A, 1978, 9, 260-262.

45 Y. Matsumi and K. Obi, Chem. Phys., 1980, 49, 87-93.

46 C. G. Cureton, K. Hara, D. V. O'Connor and D. Phillips, Chem. Phys., 1981, 63, 31-49.

47 R. S. Benten, Y. Liu and B. Abel, J. Chem. Phys., 2010, 113, 13406.

48 J. A. Davies, A. M. Green, A. M. Gardner, C. D. Withers, T. G. Wright and K. L. Reid, Phys. Chem. Chem. Phys., 2014, 16, 430-443.

49 D. S. Perry, G. A. Bethardy and X. L. Wang, Ber. Bunsen-Ges. Phys. Chem., 1995, 99, 530-535. 